EPJ Web of Conferences 106, 05004 (2016)

DOI: $10.1051 /$ epjconf/201610605004

(C) Owned by the authors, published by EDP Sciences, 2016

\title{
Improvements in Realizing 4H-SiC Thermal Neutron Detectors
}

\author{
F. Issa ${ }^{1, a}$, V. Vervisch ${ }^{1}$, L. Ottaviani ${ }^{1}$, D. Szalkai ${ }^{2}$, L.Vermeeren $^{3}$, A. Lyoussi $^{4}$, A. Kuznetsov ${ }^{5}$, \\ M. Lazar ${ }^{6}$, A. Klix ${ }^{2}$, O. Palais ${ }^{1}$, and A. Hallén ${ }^{7}$ \\ ${ }^{1}$ IM2NP (UMR CNRS 7334) - Aix-Marseille University, Case 231 -13397 Marseille Cedex 20, France \\ ${ }^{2}$ KIT- Karlsruhe Institute of Technology, Institute of Neutron Physics and Reactor Technology, \\ Karlsruhe 76344 Germany \\ ${ }^{3}$ SCK $\bullet$ CEN, Boeretang 200, 2400 Mol, Belgium \\ ${ }^{4}$ CEA, DEN, DER, Instrumentation Sensors and Dosimetry Laboratory, Cadarache, 13108 \\ St-Paul-Lez-Durance, France \\ ${ }^{5}$ University of Oslo, P.O. Box 1048 Blindern, 0316 Oslo, Norway \\ ${ }^{6}$ AMPERE (UMR 5005) - INSA de Lyon, 21 Av. Capelle, 69621 Villeurbanne, France \\ ${ }^{7}$ KTH-ICT, P.O. Box Electrum 229, 16440 Kista, Sweden
}

\begin{abstract}
In this work we presented two types of 4H-SiC semiconductor detectors (D1 and D2) both based on ion implantation of ${ }^{10} \mathrm{~B}$ inside the aluminum metallic contact. The first detector shows a high leakage current after the implantation and low signal to noise ratio. However, improvements concerning the implantation parameters and the distance between the implanted ${ }^{10} \mathrm{~B}$ thermal neutron converter layer and the active pn-junction have led to low leakage current and thus to higher signal to noise ratio. This proves the strength of this new method of realizing sensitive SiC-based thermal neutron detectors.
\end{abstract}

\section{Introduction}

In the last years semiconductor radiation detectors have received considerable attention over other types of radiation detectors thanks to their lower operating voltage, compact size, fast charge-collection times and high energy resolution. However, conventional semiconductors, such as silicon (Si) and gallium arsenide (GaAs), are still too sensitive to be used in hostile environments [1-3] where high temperature and high radiation fluxes exist. Moreover, the dark current in Si generally confines Si detector operation to room temperature operation due to the relatively large thermal generation of charge carriers at temperatures as low as $30-40^{\circ} \mathrm{C}$.

As an alternative to the conventional semiconductors, wide band gap semiconductors, such as silicon carbide ( $\mathrm{SiC}$ ) devices, have been developed for alpha particle [4-6] or X-ray detectors [7-9], and as radiation detectors in harsh environments $[10,11]$. This development is motivated by the wide band gap, high radiation resistance and high thermal conductivity of the $\mathrm{SiC}$ material.

\footnotetext{
${ }^{a}$ Corresponding author: fatima.issa@im2np.fr
}

This is an Open Access article distributed under the terms of the Creative Commons Attribution License 2.0, which permits unrestricted use, distribution, and reproduction in any medium, provided the original work is properly cited. 
$\mathrm{SiC}$ thermal neutron detectors have been realized based on juxtaposing ${ }^{6} \mathrm{LiF}$ as a neutron converter layer (NCL) on small SiC Schottky diode (200-400 $\mu$ m diameter) [12, 13], a linear counting rate with respect to the thermal neutron fluence rate was obtained. Aluminum foil was introduced between the detector and the NCL to reduce the damage within the active region which is primarily caused by the produced alpha. Moreover the thermal neutron response was unchanged by a previous irradiation to fast neutron up to $1.3 \times 10^{16} \mathrm{~cm}^{-2}$. On the other hand larger-area SiC Schottky $\left(20 \mathrm{~mm}^{2}\right)$ neutron detectors have been tested [14] based on the $100 \mu \mathrm{m}$ external ${ }^{6} \mathrm{LiF}$-based NCL. The neutron response was clearly resolvable from that of gamma, ensuring the reliability of using SiC-based detectors in environments where gamma-rays may exist $[4,15]$. Additionally another neutron detectors were realized [16, 17] based on evaporation of ${ }^{6} \mathrm{LiF}$ and ${ }^{10} \mathrm{~B}_{2} \mathrm{O}_{3}$ onto the $\mathrm{Au}$ metal contact. Such detectors showed charge collection efficiency of over $80 \%$ at self-biased voltage.

Our aim in the framework of the I_SMART project [18], is to develop an innovative complete radiation detection system based on $\mathrm{SiC}$ technology for detecting neutrons (thermal and fast) and photons in harsh environment (high temperature $\left(300-600^{\circ} \mathrm{C}\right.$ ), high pressure, and high dose rate). One part of this project is to prove the reliability of a new method of realizing thermal neutron detectors. This method is based on ion implantation of ${ }^{10} \mathrm{~B}$ into the aluminum metallic contact for converting thermal neutrons to detectable charged energetic ions. Based on this method we have previously realized the first detectors $[19,20]$ called D1. In this work we will present another type (D2 detector) which is an improvement of the D1 detector.

\section{Background}

Neutrons are uncharged and hence can travel appreciable distances in matter without interacting through a Coulomb interaction, which is the dominant mechanism through which charged particles and electrons lose their energy. Therefore mechanisms for detecting thermal neutrons are based on indirect methods where thermal neutrons interact with specific isotopes, such as ${ }^{10} \mathrm{~B}$ and ${ }^{6} \mathrm{Li}$, to react and produce charged particles. Moreover, the microscopic thermal neutron absorption cross-section of ${ }^{10} \mathrm{~B}$ is 3840 barns, leading to higher reaction probability than for ${ }^{6} \mathrm{Li}$ (940 barns) [21].

Specifically, thermal neutrons with energy of $0.025 \mathrm{eV}$ interact with ${ }^{10} \mathrm{~B}$ resulting in production of alpha particles and lithium ions, which are released in opposite directions according to the following two reactions having different probabilities:

$$
\begin{gathered}
{ }^{10} \mathrm{~B}+\mathrm{n} \rightarrow{ }^{7} \mathrm{Li}+{ }^{4} \mathrm{He}(\mathrm{Q}=2.72 \mathrm{MeV}) 6 \% \\
{ }^{10} \mathrm{~B}+\mathrm{n} \rightarrow{ }^{7} \mathrm{Li}^{*}+{ }^{4} \mathrm{He}(\mathrm{Q}=2.31 \mathrm{MeV}) 94 \% .
\end{gathered}
$$

The produced alpha and ${ }^{7} \mathrm{Li}$ charged particles generate electron-hole pairs (ehp) along their path in the semiconductor as they lose their energy by Coulomb scattering events. A rectifying junction is then created to form a space charge region supporting an electric field aiming to harvest the carriers.

\section{Fabrication Process}

We realized two types of detectors (D1 and D2) both based on a $\mathrm{p}^{+} \mathrm{n}$ junction in $4 \mathrm{H}-\mathrm{SiC}$ (see Figs. 1 and 2). For D1 detectors, the $\mathrm{p}^{+} \mathrm{n}$ diodes were fabricated with three different areas $\left(4,10\right.$ and $\left.25 \mathrm{~mm}^{2}\right)$ on commercial $\mathrm{n}^{+}$-type $4 \mathrm{H}$-Silicon Carbide wafer ( $8^{\circ}$ off-axis) purchased from CREE Research, where several epitaxial layers have been grown by chemical vapor deposition (CVD). Here we use an $80 \mu \mathrm{m}$ $\mathrm{n}$-type epitaxial layer with a low nitrogen doping concentration of $\sim 2 \times 10^{14} \mathrm{~cm}^{-3}$ covered with two p-type epitaxial layers $\mathrm{p}^{+}$and $\mathrm{p}^{++}$doped with $\mathrm{Al}$, with a thickness of $1 \mu \mathrm{m}$ each and concentrations of $2 \times 10^{17} \mathrm{~cm}^{-3}$ and $2 \times 10^{19} \mathrm{~cm}^{-3}$, respectively. The $4 \mu \mathrm{m} \mathrm{Al} \mathrm{metallic} \mathrm{contact} \mathrm{was} \mathrm{sputtered} \mathrm{at} \mathrm{room}$ temperature on the Ohmic contact metallization layer (Ni-Ti-Al-Ni alloy with $\sim 100 \mathrm{~nm}$ total thickness). 


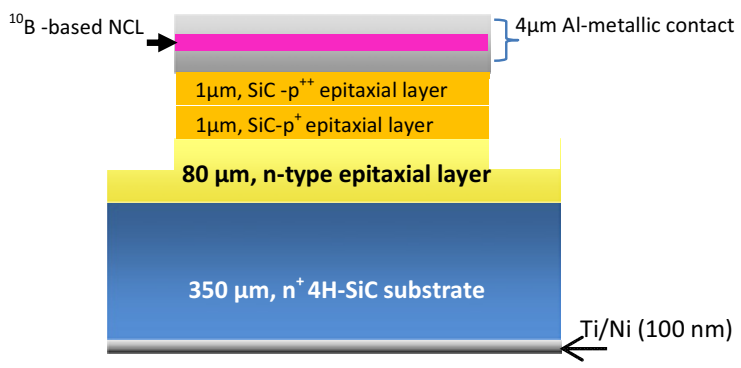

Figure 1. Schematic representation of D1 diode.

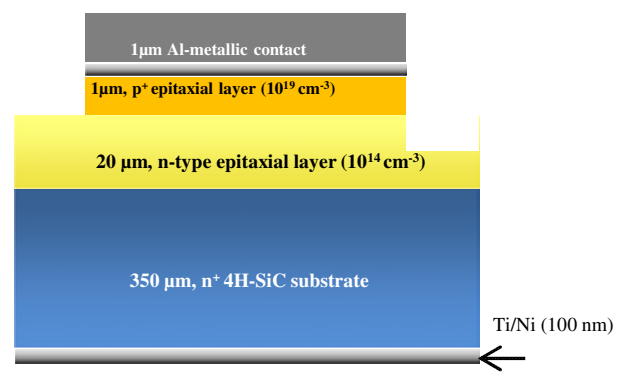

Figure 2. Schematic representation of D2 diode.

For the D2 diodes, the surface area is much smaller than the D1 diodes and Fig. 3 shows the mask that has been used to realize these D2 detectors. These diodes have been fabricated on $4 \mathrm{H}-\mathrm{SiC}{ }^{+}$ substrate purchased from Ascatron with $350 \mu \mathrm{m}$ thickness, on which n-type epitaxial layer has been grown with low doping concentration $\sim 2 \times 10^{14} \mathrm{~cm}^{-3}$. The $20 \mu \mathrm{m}$ n-epitaxial layer is covered by $1 \mu \mathrm{m}$ $\mathrm{p}^{+}$epitaxial layer with high aluminum doping concentration $\sim 10^{19} \mathrm{~cm}^{-3}$. The individual devices are then defined by etching away the $\mathrm{p}^{+}$and $\mathrm{p}^{++}$layers outside the masked areas to form mesa structures. The anode Ohmic contact (Ni/Ti/Al) with $200 \mathrm{~nm}$ diameter has been deposited by ultra-high vacuum e-beam evaporation. Finally $1 \mu \mathrm{m} \mathrm{Al}$ metallic contact has been deposited on the top of the anode Ohmic contact by e-beam evaporation.

Both types of detectors have been implanted by ${ }^{10} \mathrm{~B}$ directly into the $\mathrm{Al}$ metallic contact in order to create the NCL. The D1-diodes were implanted by ${ }^{10} \mathrm{~B}$ at $2 \mathrm{MeV}$ and a dose of $5 \times 10^{15} \mathrm{~cm}^{-2}$ at $400{ }^{\circ} \mathrm{C}$ into the thick $\mathrm{Al}$ contact layer for thermal neutron detection. Since the Al metallic contact in D2 diodes is thinner than that for D1 diodes, ${ }^{10} \mathrm{~B}$ has been implanted at lower energy $(180 \mathrm{keV})$ and a dose of $5 \times 10^{15} \mathrm{~cm}^{-2}$ in this structure. Finally both types of the $4 \mathrm{H}-\mathrm{SiC}$ diodes were fixed on alumina substrates and wire bonded to contact pads with $50 \mu \mathrm{m}$ thick wires to extract the electrical signal.

Using the SRIM software which describes the stopping and range of ions in matter [22], we estimated the profile of ${ }^{10} \mathrm{~B}$ inside the aluminum metallic contact in order to find the position of the neutron converter layer. Figures 4 and 5 show the SRIM profiles of ${ }^{10} \mathrm{~B}$ implanted into aluminum. For $\mathrm{D} 1$ diodes, the project range of ${ }^{10} \mathrm{~B}$ implanted at $2 \mathrm{MeV}$ is about $2.5 \mu \mathrm{m}$, while for the $\mathrm{D} 2$ diodes it is around $0.45 \mu \mathrm{m}$.

\section{Current Voltage Measurements}

After realizing the detectors the leakage current of each diode as function of bias voltage was measured. It is well known that the leakage current with respect to the reverse bias, which is one of the principal 


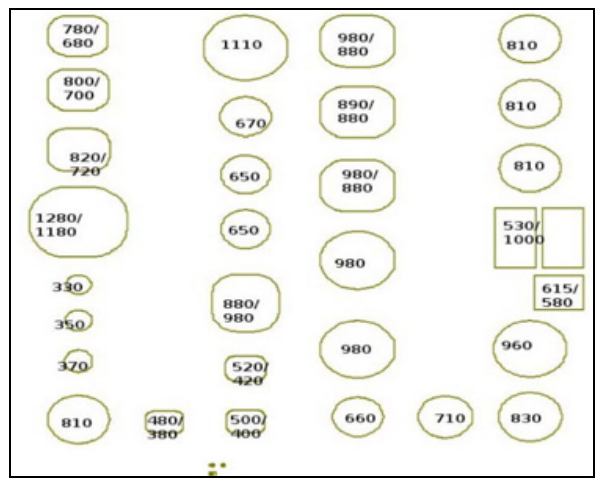

Figure 3. Picture of the pattern used to realize $\mathrm{p}^{+} \mathrm{n} \mathrm{D} 2$ diodes (the diameters are in unit of $\mu \mathrm{m}$ ).

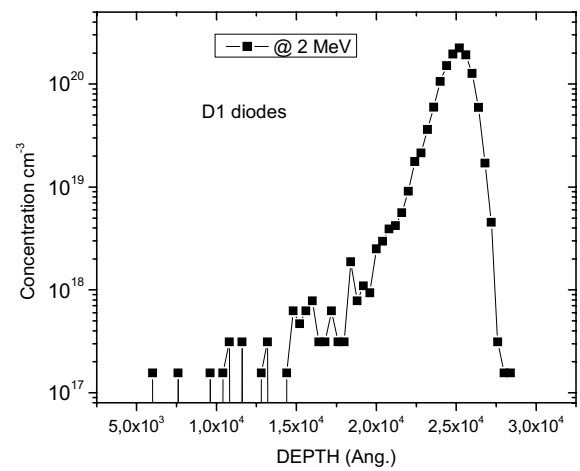

Figure 4. The distribution of ${ }^{10} \mathrm{~B}$ implanted into $\mathrm{Al}(4 \mu \mathrm{m})$ at $2 \mathrm{MeV}$ and a dose of $5 \times 10^{15} \mathrm{~cm}^{-2}$.

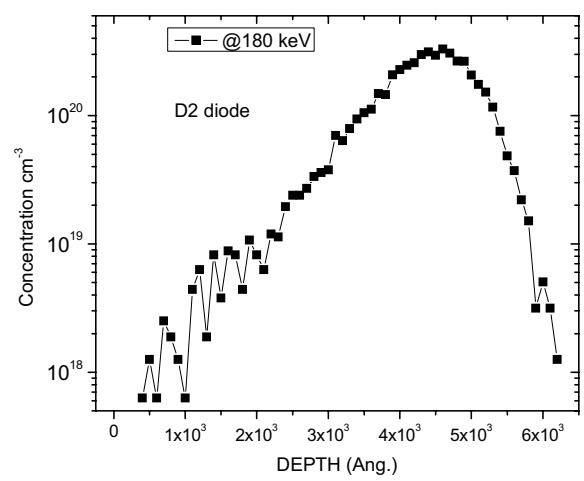

Figure 5. The distribution of ${ }^{10} \mathrm{~B}$ implanted into $\mathrm{Al}(1 \mu \mathrm{m})$ at $180 \mathrm{keV}$ and a dose of $5 \times 10^{15} \mathrm{~cm}^{-2}$.

properties of semiconductor radiation detectors, may give information about the level of defects present in the space charge region (SCR) and an estimation of the signal-to-noise ratio during the irradiation of the detectors.

The main results of the current-voltage measurement of the D1-diodes are presented in Fig. 6 . The high leakage current density $\left(10^{-4} \mathrm{~A} / \mathrm{cm}^{2}\right)$ indicates that the boron implantation at $2 \mathrm{MeV}$ at $400{ }^{\circ} \mathrm{C}$ has induced considerable amount of leakage due to the diffusion of boron beyond the $4 \mu \mathrm{m}$ Al layer. 


\section{$15^{\text {th }}$ ISRD}

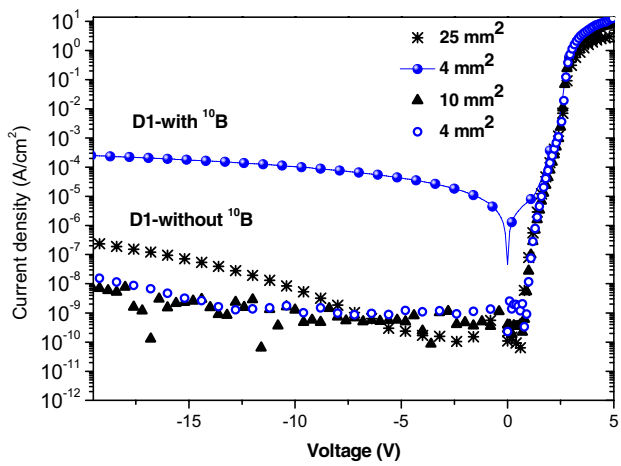

Figure 6. Current density of D1 diodes with and without ${ }^{10} \mathrm{~B}$ as function of the reverse bias.

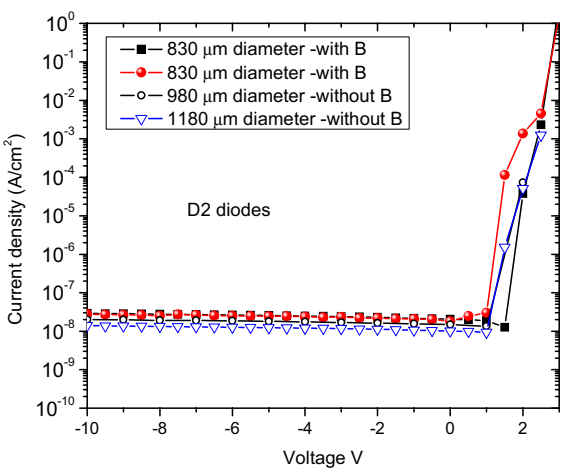

Figure 7. Current density of $\mathrm{D} 2$ diodes with and without ${ }^{10} \mathrm{~B}$ as function of the reverse bias.

Moreover, it is clear that the leakage current density increases with the diode area. This can be attributed to a higher probability of the presence of defects in the active zone for high areas. However, the D2 diodes with and without ${ }^{10} \mathrm{~B}$ show a low leakage current density around $2 \times 10^{-8} \mathrm{~A} / \mathrm{cm}^{2}$, which means that the ${ }^{10} \mathrm{~B}$ implantation process has not induced significant leakage.

\section{SRIM Simulations of Alpha and Li Ranges}

After estimating the $\mathrm{B}$ profile inside $\mathrm{Al}$, the distributions of ionization and the vacancies of both $\mathrm{Si}$ and $\mathrm{C}$ atoms caused by the charged particles $\left({ }^{4} \mathrm{He}\right.$ and $\left.{ }^{7} \mathrm{Li}\right)$ produced in the reactions have been calculated. We notice that the thickness of the SCR and its position with respect to the NCL are important parameters which should be taken into consideration while fabricating the detector, in order to collect most of the electron-hole pairs (high ionization rate region) produced by the charged particles and to minimize the radiation damage (low vacancies region) within the detector sensitive area. This can be achieved by adjusting properly the dose and the energy of implanted species.

Figures 8 and 9 show the distribution of ionizations and vacancies caused by ${ }^{7} \mathrm{Li}$ and alpha particles, assuming that they are ejected inwards, in a direction perpendicular to the device surface. As it is shown for the D1 diodes the distance between the NCL and the SCR is around $3 \mu \mathrm{m}$, which is too long for ${ }^{7} \mathrm{Li}$ ions to reach the active region. However, for the D2 diodes this distance has been reduced to around $1.35 \mu \mathrm{m}$ which is short enough so that both charged particles could contribute to the overall detector signal. 




Figure 8. The distribution of vacancies and ionization in D1 diodes caused by ${ }^{7} \mathrm{Li}$ and ${ }^{4} \mathrm{He}$ as they pass in a normal direction into the device.

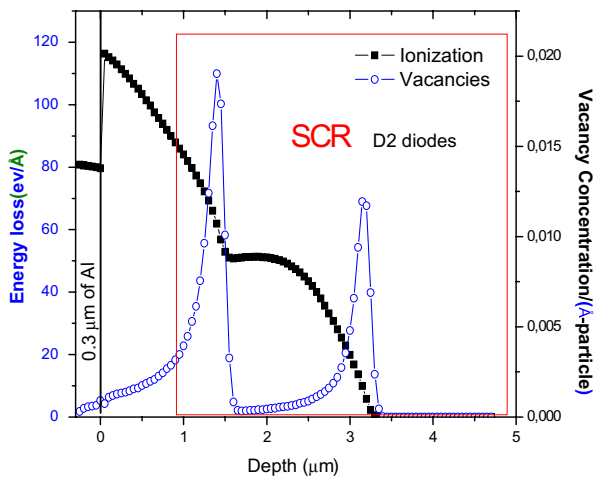

Figure 9. The distribution of vacancies and ionization in $\mathrm{D} 2$ diodes caused by ${ }^{7} \mathrm{Li}$ and ${ }^{4} \mathrm{He}$ as they pass in a normal direction into the device.

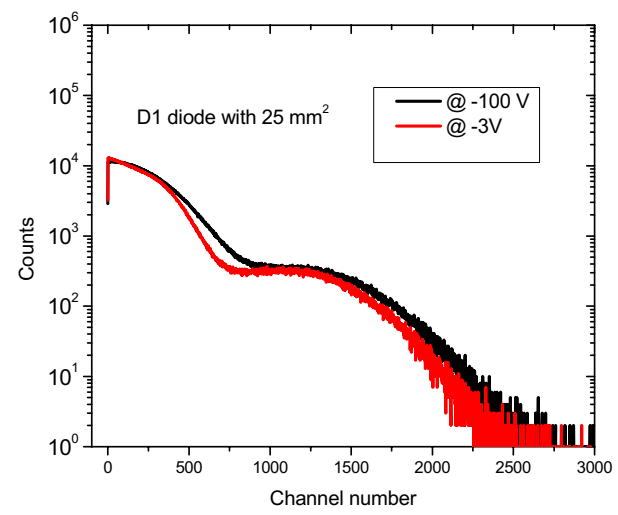

Figure 10. Thermal neutron detection spectra measured with D1-diodes with ${ }^{10} \mathrm{~B}$ based-NCL at $-3 \mathrm{~V}$ and $-100 \mathrm{~V}$ bias. 


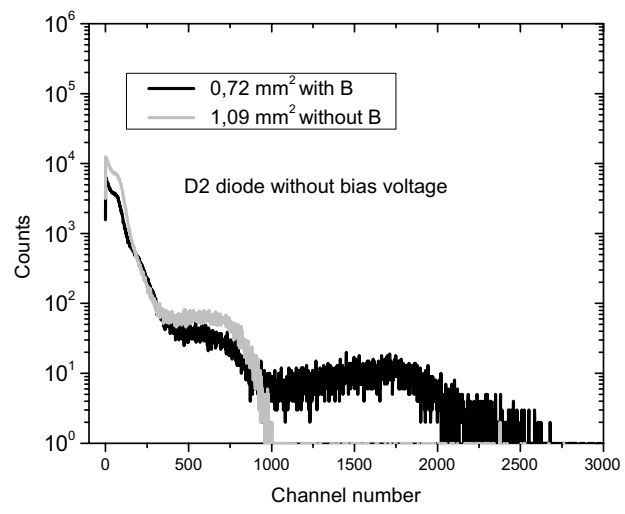

Figure 11. Thermal neutron detection spectra measured with D2-diodes with and without ${ }^{10} \mathrm{~B}$ based-NCL.

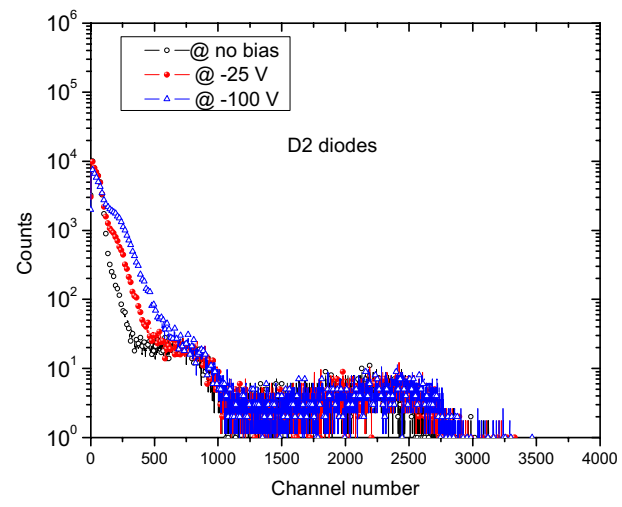

Figure 12. Thermal neutron detection spectra measured with $\mathrm{D} 2$-diodes with ${ }^{10} \mathrm{~B}$ based-NCL at zero bias, $-25 \mathrm{~V}$ and $-100 \mathrm{~V}$ bias.

\section{Neutron Irradiation Tests}

In order to study the response of the D1 and D2 detectors under thermal neutron flux, we used the facilities at the BR1 nuclear reactor at SCK.CEN in Belgium, which is a gas-cooled research reactor. It is based on natural uranium with a graphite moderator and can provide a thermal power of up to $4 \mathrm{MW}$.

We placed four diodes in one aluminum box for electromagnetic shielding. Then each diode was connected into its own coaxial cable of $3 \mathrm{~m}$ length, allowing the connection and reconnection of each diode to a charge sensitive preamplifier located on the top of the reactor. Then the aluminum box was placed into the spherical cavity of the BR1 nuclear reactor. At this location, the gamma dose rate is about $1 \mathrm{~Gy} / \mathrm{h}$, the fast neutron flux is negligible compared with the thermal neutron flux which is $7 \times 10^{8} \mathrm{n} / \mathrm{cm}^{2} \mathrm{~s}$ at $700 \mathrm{~kW}$ reactor power. The temperature is around $50{ }^{\circ} \mathrm{C}$. The diodes were irradiated at different reverse bias voltages from 0 up to $-100 \mathrm{~V}$. The output signal from the preamplifier was fed to a spectroscopic amplifier and the pulses were recorded with a multi-channel analyzer system and with a digital oscilloscope.

D1 and D2 diodes have been measured under thermal neutron fluxes (see Figs. 10 and 11, respectively). During the measurement the D1 diodes show lower signal to noise ratio compared to D2 diodes. This can be attributed to their higher leakage current. The pulse height spectra, given by D2 diodes implanted or not by ${ }^{10} \mathrm{~B}$, are shown in Fig. 11. It is obvious that $\mathrm{D} 2$ diodes with ${ }^{10} \mathrm{~B}$ show an 


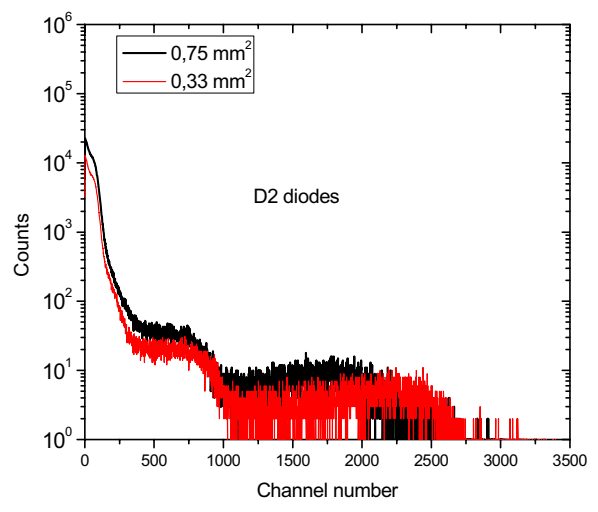

Figure 13. Thermal neutron detection spectra measured with two D2-diodes with ${ }^{10} \mathrm{~B}$ based-NCL with different areas under zero bias.

additional peak at higher channel numbers which is attributed to the detection of thermal neutrons (via alpha particles). However two other peaks still exist in diodes without ${ }^{10} \mathrm{~B}$, which are then not related to any detection of thermal neutrons. The first peak (roughly between channel numbers 100 and 500) could be attributed to gamma detection (capture gammas and gamma from aluminum decay) while the source of the second peak (around channel number 750) is still under investigation. If we compare the D1 and D2 pulse height spectra it is clear that the signal of D2 diodes is more readable, reflecting the high quality of these diodes (low leakage current and low noise level).

More measurements have been performed on D2 diodes with different areas as function of reverse biases. Figure 11 shows the pulse height spectra of D2 diode tested for 10 minutes at different bias voltages. There is no shift towards higher channel number for larger bias in the thermal neutron induced peak. This can be explained by the SRIM simulations of Fig. 8 where the SCR without external bias is already thick enough $(5.6 \mu \mathrm{m})$ to collect most of the ionization caused by the charged particles. On the contrary, the shift of the first peak into higher channel numbers with bias (as well as the increase in count rate), indicates that it is related to gamma detection. Additional measurements on D2 diodes with 0.75 and $0.33 \mathrm{~mm}^{2}$ indicate that the count rate scales with the area of the diode (Fig. 12). This confirms the sensitivity and feasibility of these detectors.

\section{Conclusions}

Two types of detectors (D1and D2) have been realized based on ion implantation of ${ }^{10} \mathrm{~B}$ inside the aluminum metallic contact. The first detector (D1) shows a low signal to noise ratio which can be attributed to its high leakage current density $\left(\sim 10^{-4} \mathrm{~A} / \mathrm{cm}^{2}\right)$ after the implantation of ${ }^{10} \mathrm{~B}$ whereas the second detectors (D2) show low leakage current and thus higher signal to noise ratio. Therefore such improvements concerning the implantation parameters and the reduced distance between the ${ }^{10} \mathrm{~B}$-based NCL and the SCR provide the strength of this new method of realizing sensitive SiC-based thermal neutron detectors.

\section{References}

[1] M. Siad, A. Keffous, Y. Belkacem, H. Menari, S. Mamma, and C. Lakhdar Chaouch, Nucl. Instruments Methods Phys. Res. Sect. A Accel. Spectrometers, Detect. Assoc. Equip. 512, 106 (2003) 
[2] F. Nava, P. Vanni, U. Biggeri, E. Vittone, C. Lanzieri, G. Bertuccio, and C. Canali, Nucl. Instruments Methods Phys. Res. Sect. A Accel. Spectrometers, Detect. Assoc. Equip. 410, 68 (1998)

[3] W. Braunschweig, T. Kubicki, K. Liibelsmeyer, D. Pandoulas, F. Tenbusch, M. Toporowsky, T. Wilms, B. Wittmer, and W. J. Xiao, Nucl. Instruments Methods Phys. Res. A 372 372, 111 (1996)

[4] F. H. Ruddy, A. R. Dulloo, J. G. Seidel, S. Seshadri, and L. B. Rowland, IEEE Trans. Nucl. Sci. 45, $536(1998)$

[5] F. Nava, P. Vanni, C. Lanzieri, and C. Canali, Nucl. Instruments Methods Phys. Res. Sect. A Accel. Spectrometers, Detect. Assoc. Equip. 437, 354 (1999)

[6] F. Ruddy, A. Dulloo, J. Seidel, J. Palmour, and R. Singh, Nucl. Instruments Methods Phys. Res. Sect. A Accel. Spectrometers, Detect. Assoc. Equip. 505, 159 (2003)

[7] G. Bertuccio, R. Casiraghi, A. Cetronio, C. Lanzieri, and F. Nava, Nucl. Instruments Methods Phys. Res. Sect. A Accel. Spectrometers, Detect. Assoc. Equip. 518, 433 (2004)

[8] G. Bertuccio, R. Casiraghi, A. Cetronio, C. Lanzieri, and F. Nava, Nucl. Instruments Methods Phys. Res. Sect. A Accel. Spectrometers, Detect. Assoc. Equip. 522, 413 (2004)

[9] F. Moscatelli, Nucl. Instruments Methods Phys. Res. Sect. A Accel. Spectrometers, Detect. Assoc. Equip. 583, 157 (2007)

[10] S. Seshadri, A. R. Dulloo, F. H. Ruddy, J. G. Seidel, and L. B. Rowland, Electron Devices, IEEE Trans. 46, 567 (1999)

[11] F. Nava, G. Bertuccio, A. Cavallini, and E. Vittone, Meas. Sci. Technol. 19, 102001 (2008)

[12] A. Dulloo, F. H. Ruddy, J. G. Seidel, J. M. Adams, J. S. Nico, and D. M. Gilliam, Nucl. Instruments Methods Phys. Res. Sect. A Accel. Spectrometers, Detect. Assoc. Equip. 498, 415 (2003)

[13] A. R. Dulloo, F. H. Ruddy, J. G. Seidel, J. M. Adams, J. S. Nico, and D. M. Gilliam, Nucl. Instruments Methods Phys. Res. Sect. A Accel. Spectrometers, Detect. Assoc. Equip. 422, 47 (1999)

[14] C. Manfredotti, A. Lo Giudice, F. Fasolo, E. Vittone, C. Paolini, F. Fizzotti, A. Zanini, G. Wagner, and C. Lanzieri, Nucl. Instruments Methods Phys. Res. Sect. A Accel. Spectrometers, Detect. Assoc. Equip. 552, 131 (2005)

[15] A. R. Dulloo, F. H. Ruddy, J. G. Seidel, C. Davison, T. Flinchbaugh, and T. Daubenspeck, IEEE Trans. Nucl. Sci. 46, 275 (1999)

[16] J. H. Ha, S. M. Kang, S. H. Park, H. S. Kim, N. H. Lee, and T.-Y. Song, Appl. Radiat. Isot. 67, 1204 (2009)

[17] J. Ha, S. Kang, S. Park, H. Kim, and Y. Kim, 355, 352 (2008)

[18] A. Lyoussi, I_SMART a Collaborative Project on Innovative Sensor for Material Ageing and Radiation Testing: European Innovative Project for SiC Applications in Harsh Media. KIC_Innoenergy,CCAV,I_SMART Proposal/Exhibit 1 (2012)

[19] F. Issa, V. Vervisch, L. Ottaviani, D. Szalkai, L. Vermeeren, A. Lyoussi, A. Kuznetsov, M. Lazar, A. Klix, O. Palais, and A. Hallén, Mater. Sci. Forum 778-780, 1046 (2014)

[20] F. Issa, V. Vervisch, L. Ottaviani, D. Szalkai, L. Vermeeren, A. Lyoussi, A. Kuznetsov, M. Lazar, A. Klix, O. Palais, and A. Hallen, IEEE Trans. Nucl. Sci. 61, 2105 (2014)

[21] G. E. Knoll, Radiation Detection and Measurement, 3rd edn (Wiley, 2000)

[22] J. F. Ziegler and J. P. Biersack, The Stopping and Range of Ions in Matter (2000) 